\title{
GEOMETRY OF NEUMANN SUBGROUPS
}

\author{
RAVI S. KULKARNI
}

(Received 25 February 1986)

Communicated by H. Lausch

\begin{abstract}
A Neumann subgroup of the classical modular group is by definition a complement of a maximal parabolic subgroup. Recently Neumann subgroups have been studied in a series of papers by Brenner and Lyndon. There is a natural extension of the notion of a Neumann subgroup in the context of any finitely generated Fuchsian group $\Gamma$ acting on the hyperbolic plane $H$ such that $\Gamma \backslash \mathbf{H}$ is homeomorphic to an open disk. Using a new geometric method we extend the work of Brenner and Lyndon in this more general context.
\end{abstract}

1980 Mathematics subject classification (Amer. Math. Soc.) (1985 Revision): 20 E 28.

\section{Introduction}

(1.1) This note essentially consists of some remarks on a series of recent papers by Brenner and Lyndon concerned with the Neumann subgroups of the classical modular group; cf. [1]-[3]. We first recall their definition. Let $\Gamma=\left\langle x, y \mid x^{2}=y^{3}=e\right\rangle$ act as the modular group on the upper half plane $\mathbf{H}$ in the standard way. Then the subgroup $P=\langle z\rangle$, where $z=x y$, is a maximal parabolic subgroup of $\Gamma$ and all such subgroups are conjugate. A subgroup $\Phi$ of $\Gamma$ is said to be non-parabolic if it contains no parabolic element. If $\Phi$ is a complement of $P$ in $\Gamma$, that is (1) $P \cap \Phi=\{e\}$, and (2) $P \cdot \Phi=\Gamma$, then $\Phi$ is called a Neumann subgroup; cf. [1]. A Neumann subgroup is maximal among non-parabolic subgroups; cf. [1, (2.8)].

This work was partially supported by the Max-Planck-Institut für Mathematik, Bonn, Germany and an NSF grant.

(C) 1989 Australian Mathematical Society $0263-6115 / 89 \$ A 2.00+0.00$ 
(1.2) In connection with these subgroups, Brenner and Lyndon were led to study transitive triples $(\Omega, A, B)$ (cf. [2]) where $\Omega$ is a countable set, $A$ and $B$ are permutations of $\Omega$ of orders 2 and 3 respectively such that the group $\langle C\rangle$, where $C=A B$, is transitive on $\Omega$. If $(\Omega, A, B)$ is a transitive triple then $\Gamma$, as in (1.1), acts on $\Omega$ in the obvious way so that the subgroup $P$ is transitive on $\Omega$. In particular $\Omega \approx \Gamma / \Phi$ for a suitable subgroup $\Phi$ whose conjugacy class is well-defined. Since $P \approx \mathbf{Z}$ it is clear that either $P$ acts simply transitively on $\Omega$ in which case $\Omega$ is an infinite set, or else $P$ acts ineffectively on $\Omega$ in which case $\Omega$ is a finite set. In the first case $\Phi$ is a Neumann subgroup. In the second case $(\Gamma: \Phi)<\infty$ and $\Phi \backslash H$ has only one cusp. Such a subgroup was called cycloidal by Petersson [9]. Thus the study of transitive triples amounts to a simultaneous study of Neumann and cycloidal subgroups of $\Gamma$. For the earlier work on Neumann subgroups see [8], [6], [13], and also [7, pages 119-122].

(1.3) A principal result in [1] which extends Theorem 2 of [13] is a structure-and-realization theorem for Neumann subgroups. Similar and more general results were proved by Stothers [10]-[12]. The proof in [1] is based on a correspondence between transitive triples and Eulerian paths in cuboid graphs, that is, the graphs with vertex-valences at most 3 . For the triples associated with torsion-free Neumann-or-cycloidal subgroups the correspondence is one-to-one, but in general to make the correspondence one-to-one one would need to put an extra structure on the cuboid graphs. The same method is used in [3] to produce maximal-among-non-parabolic subgroups which are not Neumann.

(1.4) In this note we extend this work to

$$
\Gamma=\prod_{i=1}^{* n} \Gamma_{i}, \quad \Gamma_{i}=\left\langle x_{i}\right\rangle \approx \mathbf{Z}_{m_{i}}, \quad 2 \leq m_{i}, n<\infty .
$$

Except for $\Gamma \approx \mathbf{Z}_{2} * \mathbf{Z}_{2}$, these groups can be realized as discrete subgroups of the orientation-preserving isometries of the hyperbolic plane $\mathbf{H}$ such that $\Gamma \backslash \mathbf{H}$ has finite area, and $x_{i}$ acts as a rotation through angle $2 \pi / m_{i}$ around its fixed point. Then the element $u=x_{1} x_{2} \cdots x_{n}$ is parabolic.

(1.5) The above remarks are meant only for motivation. In the following, hyperbolic geometry will not be used explicitly. We start with $\Gamma$ as in (1.4.1). Let $u=x_{1} \cdots x_{n}$. The conjugates of $u^{k}, k \neq 0$ are called the parabolic elements of $\Gamma$. Let $P=\langle u\rangle$. A subgroup of $\Gamma$ is called parabolic if all of its non-identity elements are parabolic. Clearly the maximal parabolic subgroups are precisely the conjugates of $P$. A subgroup of $\Gamma$ is called nonparabolic if it contains no parabolic element. A complement $\Phi$ of $P$ in $\Gamma$ is called a Neumann subgroup. Thus, for a Neumann subgroup $\Phi$ one has (i) $P \cap \Phi=\{e\}$, and (ii) $P \cdot \Phi=\Gamma$. The latter implies (ii) $|P \backslash \Gamma / \Phi|=1$. 
Conversely, if (ii) ${ }^{\prime}$ holds and $(\Gamma: \Phi)=\infty$ then $\Phi$ is a Neumann subgroup. If (ii) holds and $(\Gamma: \Phi)<\infty$ then as in [5], $\Phi$ is called a 1-cycloidal subgroup. (In the correspondence between subgroups of the Fuchsian groups and holomorphic maps among Riemann surfaces, the 1-cycloidal subgroups precisely correspond to meromorphic functions on closed Riemann surfaces with a single pole; these functions may be considered as generalizations of polynomial maps; cf. [5].) One sees (cf. (2.1)) that a Neumann subgroup is maximal among non-parabolic subgroups.

(1.6) Let $\Gamma$ be as in (1.4.1). For $\Phi \leq \Gamma$, in [4] we attached a diagram $X_{\Phi}$ and its thickening $\mathbf{X}_{\Phi}$ with canonical projections $X_{\Phi} \rightarrow X_{\Gamma}, \mathbf{X}_{\Phi} \rightarrow \mathbf{X}_{\Gamma}$. Here $\mathbf{X}_{\boldsymbol{\Phi}}$ is an orientable surface with non-empty boundary $\partial \mathbf{X}_{\Phi}$. One may think of $\mathbf{X}_{\Gamma}$ as " $\Gamma \backslash \mathbf{H}$ with the cusp cut off". This makes the "cuspidal infinity" more tangible-for example, one gets the following useful characterizations: $\Phi \leq \Gamma$ is Neumann (respectively 1 -cycloidal) if and only if $\partial \mathbf{X}_{\Phi}$ is connected and non-compact (respectively connected and compact). Pinching each circle in $X_{\Phi}$ to a point one obtains a graph $Y_{\Phi}$ whose structure suggests the notion of an $\left(m_{1}, \ldots, m_{n}\right)$-semiregular graph; cf. (2.4). If $\Phi$ is a Neumann subgroup then the image of $\partial \mathbf{X}_{\Phi}$ in $Y_{\Phi}$ is a special type of Eulerian path which we simply call admissible. This provides a natural explanation of the initially intriguing Brenner-Lyndon correspondence between Neumann and 1-cycloidal subgroups of the modular group and the Eulerian paths in cuboid graphs. A natural extension of their results is as follows: the conjugacy classes of Neumann (respectively 1-cycloidal) subgroups of $\Gamma$ are in one-to-one correspondence with the admissible Eulerian paths in $\left(m_{1}, \ldots, m_{n}\right)$-semiregular graphs.

(1.7) For $\Gamma$ as in (1.4.1) and $\Phi \leq \Gamma$ we have by Kurosh's theorem,

$$
\Phi \approx F_{r} * \prod_{i=1}^{* n}\left(\prod_{j \in J_{i}}^{*} \Phi_{i j}\right),
$$

where $F_{r}$ denotes the free group of rank $r$ and $\Phi_{i j} \cong \mathbf{Z}_{d}, d \mid m_{i}$, are conjugates to subgroups of $\Gamma_{i}$. In (1.7.1) we assume that $\Phi i j \not\{\}$ with the understanding that if $J_{i}$ is empty then $\prod_{j \in J_{i}}^{*} \Phi_{i j}=\{e\}$. Let

$$
r_{i}(d)=\#\left\{\Phi_{i j}=\mathbf{Z}_{m_{i} / d}\right\}, \quad d \mid m_{i}, d<m_{i} .
$$

The numbers $r_{i}(d)$ may be possibly infinite. In Section 4 we prove a structureand-realization theorem for Neumann subgroups. For example, if at most one $m_{i}$ is even, then $\Phi$ as in (1.7.1) is realizable as a Neumann subgroup if and only if either (1) $r=\infty$ or (2) $r$ is an even integer and $r_{i}(1)=\infty$ for at least $n-1$ values of $i$. If there are two even $m_{i}$ 's there is a curious new family of Neumann subgroups (cf. (2.11)) of which there is no analogue in the case of the modular group. This family makes the full structure theorem a 
bit complicated, but the underlying geometric idea is simple. For details see Section 4.

(1.8) Finally in Section 5 we give some geometric constructions of subgroups which are maximal, or maximal with respect to some additional properties such as Neumann, 1-cycloidal, non-parabolic but non-Neumann,....

I wish to thank W. W. Stothers for drawing my attention to [2].

\section{Preliminaries}

(2.0) Throughout this section let $\Gamma, \Gamma_{i}, x_{i}, u$ be as in (1.4) and (1.5). We use the terminology introduced there.

(2.1) Proposition. A Neumann subgroup is maximal among non-parabolic subgroups.

Proof. Let $\Phi$ be a Neumann subgroup of $\Gamma$, and $P=\langle u\rangle$. So $P$ acts simply transitively on $\Gamma / \Phi$. The isotropy subgroup of $P$ at $a \Phi$ is $P \cap a \Phi a^{-1}=\{e\}$. So $a^{-1} P a \cap \Phi=\{e\}$, that is, $\Phi$ is a non-parabolic subgroup. If $\Psi$ is a subgroup of $\Gamma$ which properly contains $\Phi$ then $P$ acts transitively but not simply transitively on $\Gamma / \Psi$. But since $P \approx \mathrm{Z}$, this means that the $P$-action on $\Gamma / \Psi$ is ineffective and $|\Gamma / \Psi|<\infty$. Hence $P \cap \Psi \neq\{e\}$, that is, $\Psi$ contains parabolic elements. So $\Phi$ is maximal among non-parabolic subgroups.

(2.2) As in [4], let $X_{\Gamma}$ be a diagram for $\Gamma$ and $X_{\Gamma}$ its thickening

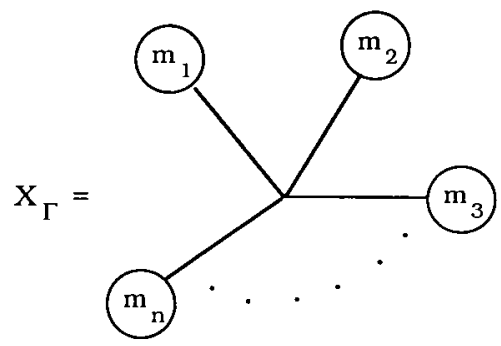

$(2.2 .1)$

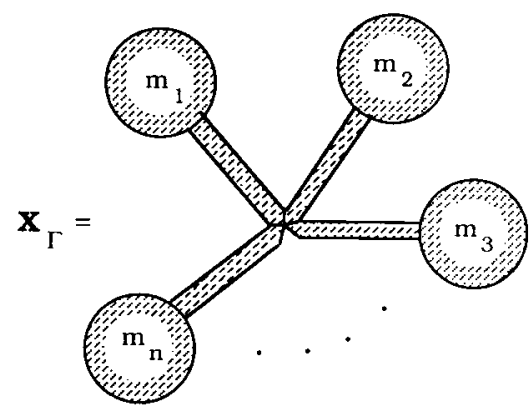


In the thickening each circle with $m<\infty$ is replaced by a disk and each segment is replaced by a rectangular sheet. These pieces are attached as shown in the figure so as to form a compact orientable surface with boundary. The case of a circle with $m=\infty$ does not occur in this paper. In that case a circle would be replaced by an annulus.

A building block of type $i$ has the form

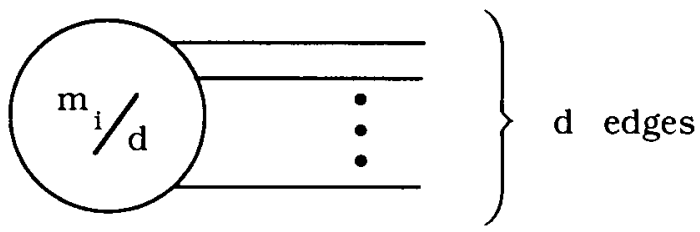

and is denoted by $B_{i}(d)$. A diagram $X_{\Phi}$ is built out of such $B_{i}(d)$ 's and there is a canonical projection $X_{\Phi} \rightarrow X_{\Gamma}$. The thickening of $B_{i}(d)$ is

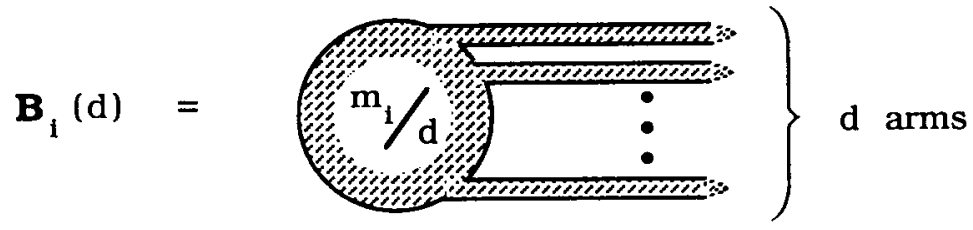

The thickening $\mathbf{X}_{\Phi}$ of $X_{\Phi}$ is built out of $\mathbf{B}_{i}(d)$ 's. Notice that $\mathbf{X}_{\Phi}$ is an orientable surface with boundary $\partial \mathbf{X}_{\Phi}$. There is a canonical projection $p: \mathbf{X}_{\Phi} \rightarrow$ $\mathbf{X}_{\Gamma}$ and also a "thinning" map $\mathbf{X}_{\Phi} \rightarrow X_{\Phi}$ (the restriction $\left.p\right|_{\text {int }} \mathbf{X}_{\Phi}$ : int $\mathbf{X}_{\Phi} \rightarrow$ int $\mathbf{X}_{\Gamma}$ is a branched covering of surfaces; if $\Gamma$ is realized as an orientationpreserving, properly discontinuous group of homeomorphisms of $\mathbf{R}^{2}$ then $\left.p\right|_{\text {int }} \mathrm{X}_{\Phi}$ is equivalent to the canonical map $\left.\Phi \backslash \mathbf{R}^{2} \rightarrow \Gamma \backslash \mathbf{R}^{2}\right)$. The shape of $\mathbf{B}_{i}(d)$ may be described as "a closed disk with $d$ arms." Each of the dotted edges at the end of an arm is its half-outlet; together they form an outlet. In $\mathbf{X}_{\Phi}$ the outlets come in groups of $n$. So we may use the obvious and suggestive terminology of an angle formed by the half-outlets. For example the interior angle formed by the half-outlets of an arm is $2 \pi / n$. In $X_{\Phi}$ one half-outlet of an arm of $\mathbf{a} \mathbf{B}_{i}(*)$ is joined at half-outlet of $\operatorname{arm}$ of $\mathbf{B}_{i+1}(*)$, where the subscript $i$ is counted $\bmod n$. In the sequel, it will be important to keep in mind that

$$
\partial \mathbf{B}_{i}(d)=\left\{\mathbf{B}_{i}(d) \cap \partial \mathbf{X}_{\Phi}\right\} \cup\{\text { the outlets }\} .
$$

(2.3) Pinching each circle in $X_{\Phi}$ to a point one gets a graph $Y_{\Phi}$. Again one has a canonical projection denoted by $p: Y_{\Phi} \rightarrow Y_{\Gamma}$. Notice that the terminal vertices, that is, the vertices of valence 1 , of $Y_{\Phi}$ are precisely the images of $m_{i}$ 
in $X_{\Phi}$. The vertices adjacent to terminal vertices will be called sub-terminal vertices. Now

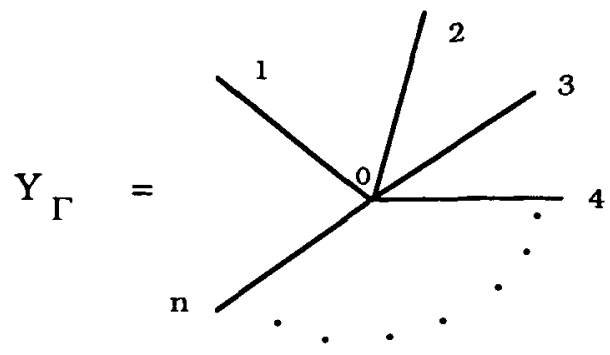

has $n+1$ vertices-the image of $m_{i}$ is numbered $i$, and the "base-vertex" is numbered 0 . So the vertices of $Y_{\Phi}$ are divided into $n+1$ disjoint subsets:

$$
\alpha_{i}=\{v \mid p(v) \text { has number } i\} .
$$

The structure of $Y_{\Phi}$ motivates the following

(2.4) Definition. Let $n$ and $m_{1}, \ldots, m_{n}$ be positive integers, each at least 2. An $\left(m_{1}, \ldots, m_{n}\right)$-semiregular graph is a connected graph $G$ whose vertices are divided into $n+1$ disjoint subsets $\alpha_{i}, i=0,1, \ldots, n$, such that

(a) $v \in \alpha_{i}$ implies valence $v=n$ (respectively a divisor of $m_{i}$ ) if $i=0$ (respectively if $i \geq 1$ ),

(b) each edge of $G$ has one end in $\alpha_{0}$ and the other in $\alpha_{i}, i \geq 1$,

(c) given $v \in \alpha_{0}$ and $i \geq 1$, there is a unique edge joining $v$ to a vertex in $\alpha_{i}$.

Clearly $Y_{\Phi}$, as in (2.3), is an $\left(m_{1}, \ldots, m_{n}\right)$-semiregular graph.

If $n=2$ (respectively some $m_{i}$ is even), then the vertices in $\alpha_{0}$ (respectively certain vertices in $\alpha_{i}$ ) have valence 2 , and if convenient may well be not counted as vertices. Thus for example, not counting the vertices in $\alpha_{0}$, an $\left(m_{1}, m_{2}\right)$-semiregular graph is a bipartite graph. Again if $G$ is a $(2, k)$ semiregular graph such that all vertices in $\alpha_{1}$ (respectively $\alpha_{2}$ ) have valence 2 (respectively $k$ ), then not counting the vertices either in $\alpha_{0}$ or in $\alpha_{1}$, one has a $k$-regular graph in the usual sense. Thus if $\Gamma=\mathbf{Z}_{2} * \mathbf{Z}_{k}$, and $\Phi \leq \Gamma$ is torsion-free then $Y_{\Phi}$ may be considered as a $k$-regular graph. In particular, corresponding to torsion-free subgroups of the modular group one gets cubic graphs.

(2.5) Remark. Let $G$ be an $\left(m_{1}, \ldots, m_{n}\right)$-semiregular graph. Then the edges at a vertex in $\alpha_{0}$ come equipped with a natural cyclic order. Now suppose at each vertex $v \in \alpha_{i}, i \geq 1$, we specify some cyclic order among the edges incident with $v$. Then we may replace each $v \in \alpha_{i}, i \geq 1$, by a circle and attach the $v$-ends of the edges incident at $v$ to the circle consistent 
with the prescribed cyclic order, and obtain a diagram $X$. This diagram has a canonical projection $p: X \rightarrow X_{\Gamma}$; see the proof of [4, Theorem 1]. $X$ may be considered as $X_{\Phi}$ for a subgroup $\Phi$ whose conjugacy class is well defined. Thus $G$ is isomorphic to $Y_{\Phi}$ for some $\Phi \leq \Gamma$.

(2.6) Taking a base point in $\partial \mathbf{X}_{\Gamma}$ we may represent $u=x_{1} \cdots x_{n}$ by the oriented boundary $\partial \mathbf{X}_{\Gamma}$. Now $p^{-1}\left(\partial \mathbf{X}_{\Gamma}\right)=\partial \mathbf{X}_{\Phi}$, so the components of $\mathbf{X}_{\Phi}$ are in one-to-one correspondence with the double cosets $P \backslash \Gamma / \Phi$. If $C$ is a component of $\partial \mathbf{X}_{\phi}$ and $\left.P\right|_{C}: C \rightarrow \partial \mathbf{X}_{\Gamma}$ has degree $d$ (possibly infinite), then $d$ is the number of points in the corresponding $P$-orbit in $\Gamma / \Phi$. In particular $C$ is non-compact if and only if $d=\infty$, which is if and only if the $P$-action on the corresponding orbit is effective. Clearly one gets

PROPOSITION. (1) $\Phi$ is a non-parabolic subgroup if and only if $\partial \mathbf{X}_{\Phi}$ has no compact component.

(2) $\Phi$ is a Neumann (respectively 1-cycloidal) subgroup if and only if $\partial \mathbf{X}_{\Phi}$ is connected and non-compact (respectively connected and compact).

(2.7) We recall some elementary facts from the topology of surfaces. Let $M$ be any connected surface possibly with non-empty boundary. A connected, compact subsurface $S$ of $M$ is said to be tight if $M$ - int $S$ has no compact component. Notice that if $S$ is a compact subsurface then $M-S$ has only finitely many components. So if $S$ is compact and connected then $S_{1}=$ $S \cup\{$ compact components of $M-$ int $S\}$ is a tight subsurface. It is now clear that $M$ admits an exhaustion by tight subsurfaces, that is, a sequence $S_{i}$, $i=1,2, \ldots$, of tight subsurfaces such that $S_{i} \subseteq$ int $S_{i+1}$ and $M=\bigcup_{i} S_{i}$.

Now suppose that the fundamental groups of $M$ based at $*$ is finitely generated. So there exist finitely many based loops $C_{i}$ such that $\pi_{1}(M, *)=$ $\left\langle\left[C_{i}\right]\right\rangle$, where $\left[C_{i}\right]$ denotes the homotopy class of $C_{i}$. One says that an arcconnected subset $A$ of $M$ carries $\pi_{1}$ if the canonical map $\pi_{1}(A) \rightarrow \pi_{1}(M)$ is surjective. Clearly any arc-connected subset $A$ containing $\cup C_{i}$ carries $\pi_{1}$. Now let $S$ be a tight subsurface which contains $\cup C_{i}$. In this case in fact the canonical map $\pi_{1}(S) \rightarrow \pi_{1}(M)$ is an isomorphism and it is easy to see that each component of $M-S$ is either a cylinder or a disk. If $\partial M \neq \varnothing$ these cylinders or disks may also have non-empty boundary.

(2.8) We apply the considerations in (2.7) to $\mathbf{X}_{\Phi}$. Let $S$ be a tight subsurface of $\mathbf{X}_{\Phi}$. Then for each building block $\mathbf{B}_{i}(d)$ we see that a component of $S \cap \mathbf{B}_{i}(d)$ is also tight. Let $S_{1}$ be the union of $S$ and all $\mathbf{B}_{i}(d)$ 's which intersect $S$ in a subset with non-empty interior. Then $S_{1}$ has the additional property

$$
\partial S_{1}=\left(S_{1} \cap \partial \mathbf{X}_{\Phi}\right) \cup A,
$$

where $A$ is the union of the half-outlets on some arms of the building blocks. Now suppose $\Phi$ is as in (1.7.1). Then its free part $F_{r}$ may be identified with 
$\pi_{1}\left(Y_{\Phi}\right)$ or $\pi_{1}\left(\mathbf{X}_{\Phi}\right)$ (see the discussion in [5, Section 2]. Suppose that $r<\infty$. So there exist tight subsurfaces of $\mathbf{X}_{\Phi}$ which carry $\pi_{1}$; cf. (2.7). We call a tight subsurface characteristic if it carries $\pi_{1}$ and has the additional property stated in (2.8.1).

From the above discussion it is clear that if $r<\infty, \mathbf{X}_{\Phi}$ admits an exhaustion by characteristic subsurfaces.

(2.9) Proposition. Let $\Phi$ as in (1.7.1) be a Neumann subgroup of $\Gamma$ and $r<\infty$. Let $S$ be a characteristic subsurface of $\mathbf{X}_{\Phi}$. Then $\partial S$ is connected and contains exactly one pair of half-outlets making an exterior angle $2 \pi / n ; c f$. (2.2). Moreover $\operatorname{int}\left(\mathbf{X}_{\Phi}-S\right)$ is homeomorphic to an open disk and $\partial\left(\mathbf{X}_{\Phi}-S\right)$ has two components, each homeomorphic to an open interval.

Proof. Since $S$ is characteristic, we have $\partial S=\left\{S \cap \partial \mathbf{X}_{\Phi}\right\} \cup A$, where $A$ is a union of half-outlets. Since $\partial S$ is compact, $\partial \mathbf{X}_{\boldsymbol{\Phi}}$ is connected and noncompact (cf. (2.6)) we see that each component of $\partial S$ must intersect $A$ as well as $\partial \mathbf{X}_{\Phi}$. Notice that the half-outlets in $A$ come in pairs-each pair forms a connected arc, and different pairs form disjoint arcs.

First we claim that $\partial S$ is connected. Suppose $C_{1}, C_{2}$ are two disjoint components of $\partial S$. Since $C_{1}, C_{2}$ contain points of $\partial \mathbf{X}_{\Phi}$, and $\partial \mathbf{X}_{\Phi}$ is connected, there is an $\operatorname{arc} \varepsilon \subseteq \partial \mathbf{X}_{\Phi}$ joining a point $p_{1}$ in $C_{1}$ to a point $p_{2}$ in $C_{2}$. But since $S$ is connected there is an arc $\beta \subseteq S$ joining $p_{1}$ to $p_{2}$ and passing though a base-point *. But then $\alpha \cup \beta$ forms a based loop whose homotopy class is clearly not contained in $\pi_{1}(S, *)$. This would contradict that $S$ carries $\pi_{1}$. So $\partial S$ is connected.

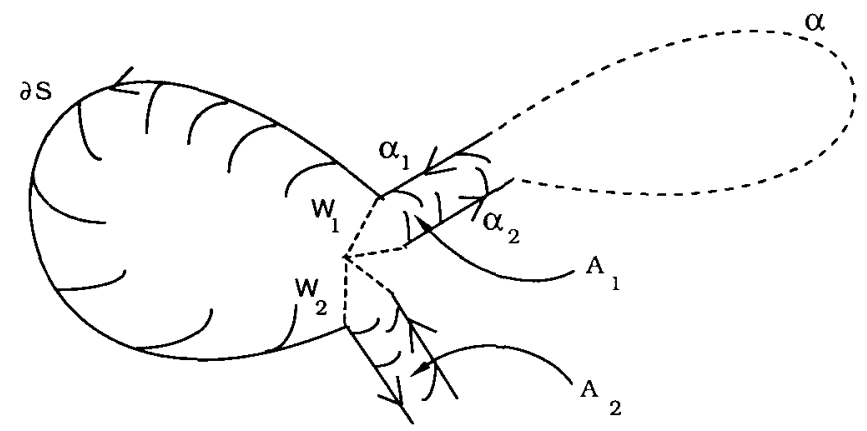

Next suppose, if possible, that there are two pairs of half-outlets, each pair forming an arc. Then $\partial S-A$ has two components which must be connected by an arc $\subseteq \partial \mathbf{X}_{\Phi}$, and we get a contradiction exactly as above.

Next suppose that the pair $w_{1}, w_{2}$ of half-outlets makes an exterior angle strictly greater than $2 \pi / n$ (see the figure in (2.9.1)). Then the arms $A_{1}, A_{2}$ in 
$\mathbf{X}_{\Phi}-$ int $S$ incident with $w_{1}$ and $w_{2}$ are distinct. Again since $\partial \mathbf{X}_{\Phi}$ is connected, the components $\alpha_{1}, \alpha_{2}$ of $A_{1} \cap \partial \mathbf{X}_{\Phi}$ are joined by an arc $\alpha \subseteq \partial \mathbf{X}_{\Phi}$. Clearly $\alpha \cap S=\varnothing$. Now $\partial A_{1} \cup \alpha$ forms a Jordan curve outside $S$. Since $S$ carries $\pi_{1}$ this Jordan curve must bound a disk. But then $\mathbf{X}_{\Phi}-$ int $S$ would have a compact component and $S$ would not be tight. This contradiction shows that the exterior angle formed by $w_{1}, w_{2}$ must be $2 \pi / n$, and so $w_{1} \cup w_{2}$ is an outlet of an arm lying outside int $S$. This arm connects $\mathrm{x}_{\Phi}-$ int $S$ to $S$. In particular $\mathbf{X}_{\Phi}-$ int $S$ has only one component. From the remarks in (2.7) it is now clear that int $\left(\mathbf{X}_{\Phi}-\right.$ int $\left.S\right)$ is homeomorphic to a disk and $\partial\left(\mathbf{X}_{\Phi}-\right.$ int $\left.S\right)$ has two components each sharing one endpoint of $\partial S-A$.

(2.10) The above proposition may be used to get an intuitive understanding of a Neumann subgroup $\Phi$ with $r<\infty$. Let $S_{1} \subset S_{2} \subset \cdots$ be an exhaustion of $\mathbf{X}_{\Phi}$ by characteristic subsurfaces. Each $S_{k+1}-$ int $S_{k}$ is homeomorphic to a closed disk. Also each $S_{k}$ has exactly one pair of half-outlets with exterior angle $2 \pi / n$. Inserting an appropriate $B_{i}(1)$ in this outlet we obtain a new diagram $\widetilde{S}_{k} \approx \mathbf{X}_{\Phi_{k}}$ where $\Phi_{k}$ is a 1-cycloidal subgroup. Thus we get a sequence $\Phi_{k}, k=1,2, \ldots$, of 1-cycloidal groups so that $\mathbf{X}_{\Phi_{k}}$ contains some $\mathbf{B}_{i}(1)$ and $\mathbf{X}_{\Phi_{k+1}}$ is obtained from $\mathbf{X}_{\Phi_{k}}$ by removing some $\mathbf{B}_{i}(1)$, and inserting some $\mathbf{B}_{i}(d), d>1$, together with some outer building blocks so that the union of the newly inserted building blocks is a subset homeomorphic to a closed disk. We express this by saying that $\Phi$ is obtained by unfolding a sequence of 1-cycloidal subgroups $\boldsymbol{\Phi}_{k}$.

(2.11) We shall now describe a special "unfolding" of a single 1-cycloidal subgroup. It will be important in the structure theory of Neumann subgroups in Section 4. Suppose we have two $m_{i}$ 's, say $m_{a}, m_{b}$, which are even integers. Let $\Phi_{0}$ be a 1-cycloidal subgroup so that $X_{\Phi_{0}}$ contains either $B_{a}(1)$ or $B_{b}(1)$, say the first. Then we can obtain a Neumann subgroup $\Phi$ as follows, which is best described by its diagram $X_{\Phi}$.

Suppose

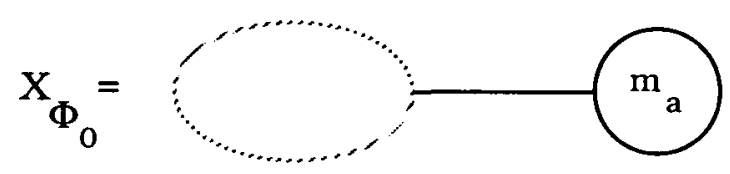

Let

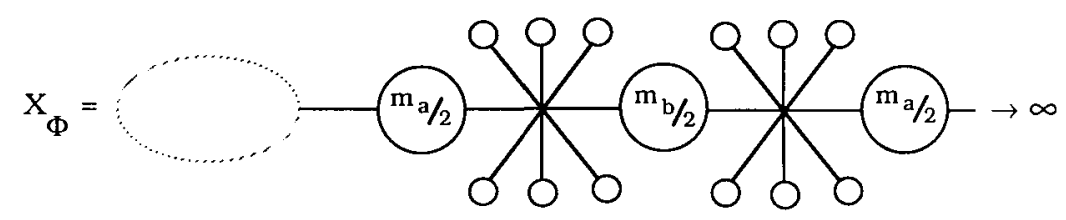


Here all the unlabelled building blocks in the newly inserted portion are $B_{i}(1)$ 's, $i \neq a, b$. We shall say that $\Phi$ is a simple $\left(m_{a}, m_{b}\right)$-unfolding of $a$ 1-cycloidal subgroup $\Phi_{0}$.

(2.12) Remark. Let $\Phi$ as (2.7.1) be a Neumann subgroup with $r=\infty$. Then $\mathbf{X}_{\Phi}$ contains no characteristic subsurface. But it is not difficult to see that still $\mathbf{X}_{\Phi}$ admits an exhaustion $S_{k}, k=1,2 \ldots$, by tight subsurfaces which satisfy the property stated in (2.8.1) and such that $\partial S_{k}$ may contain several pairs of half-outlets. Filling these pairs by suitable $\mathbf{B}_{i}(1)$ 's we obtain $\widetilde{S}_{k} \approx \mathbf{X}_{\Phi_{k}}$ where $\Phi_{k}$ is a 1-cycloidal subgroup. In this sense $\Phi$ can still be considered as an "unfolding of a sequence of 1-cycloidal subgroups."

\section{Eulerian paths}

Let $G$ be a graph. Each edge of $G$ can be directed in two ways and so corresponds to two directed edges, each of which is the inverse of the other. A path in $G$ is reduced if it contains no consecutive pair of inverse edges. An Eulerian path in $G$ is a path which contains each directed edge once and which is reduced except at the terminal vertices.

(3.2) Let $G$ be a $\left(m_{1}, \ldots, m_{n}\right)$-semiregular graph; cf. (2.4). An admissible path in $G$ is a path in which the vertices occur in the following consecutive order:

$$
\cdots v_{1} w_{1} v_{2} w_{2} \cdots, \quad v_{i} \in \alpha_{0}, w_{i} \in \alpha_{k+i},
$$

where $k$ is some fixed integer and $\alpha_{k+i}=a_{j}$, where $j$ is the unique positive integer such that $1 \leq j \leq n, k+i=j(n)$.

(3.3) THEOREM. Let $\Gamma$ be as (1.4.1). Then the conjugacy of Neumann (respectively 1-cycloidal) subgroups of $\Gamma$ are in one-to-one correspondence with the admissible Eulerian paths in infinite (respectively finite) $\left(m_{1}, \ldots, m_{n}\right)$ semiregular graphs.

Proof. Let $\Phi$ be a Neumann (respectively 1-cycloidal) subgroup $\Gamma$. Then $Y_{\Phi}$ is an $\left(m_{1}, \ldots, m_{n}\right)$-semiregular graph. Since $\Phi$ is Neumann (respectively 1-cycloidal), $Y_{\Phi}$ is infinite (respectively finite). Now orient $\mathbf{X}_{\Phi}$; this also orients $\partial \mathbf{X}_{\Phi}$. If $A$ is an arm of a building block of $\mathbf{X}_{\Phi}$ then $A \cap \partial \mathbf{X}_{\Phi}$ consists of two edges, which, under the canonical projection $\mathbf{X}_{\Phi} \rightarrow X_{\Phi} \rightarrow Y_{\Phi}$, project onto a pair of mutually inverse directed edges. It follows that the image of $\partial \mathbf{X}_{\Phi}$ in $Y_{\Phi}$ is an admissible Eulerian path. 
Conversely, let $G$ be an infinite (respectively finite) $\left(m_{1}, \ldots, m_{n}\right)$-semiregular graph, and $E$ an admissible Eulerian path in $G$. Let $v \in \alpha_{i}, i \geq 1$. Introduce a cyclic order among the (undirected) edges incident with $v$ as follows: an edge $f$ cyclically follows $e$ if and only if in $E$ the directed edge $e$ ending in $v$ follows the directed edge $f$ beginning at $v$. By the remark in (2.5) we can construct an infinite (respectively finite) diagram $X$ which corresponds to a conjugacy class of a subgroup $\Phi$. But the existence of $E$ also shows that $\partial \mathbf{X}$ is connected and is non-compact (respectively compact) so $\Phi$ is Neumann (respectively 1-cycloidal).

It is easy to see that this establishes the one-to-one correspondence asserted in the theorem.

\section{A structure theorem}

(4.0) Throughout this section $\Gamma$ is as in (1.4.1) and $\Phi$ is as in (1.7.1) and we use the notation used there. If $\Gamma \approx \mathbf{Z}_{2} * \mathbf{Z}_{2}$ it is easy to see that the two conjugacy classes of subgroups isomorphic to $\mathbf{Z}_{2}$ precisely consist of all the Neumann subgroups in $\Gamma$. Henceforth we shall assume that $\Gamma \not \mathbf{Z}_{2} * \mathbf{Z}_{2}$.

(4.1) Proposition. If $r=\infty$ then $\Phi$ is realizable as a Neumann subgroup.

Proof. The details of this proof are similar to (and simpler than) those of [5, Theorem (1.5)], which deals with the case of 1-cycloidal subgroups. So we shall be brief. First of all, the diophantine condition (see [5, (3.2)]), needed there is no longer necessary since the "difficulties can be thrown off to infinity." Recall that for $d \mid m_{i}, d<m_{i}$

$$
r_{i}(d)=\#\left\{\phi_{i j} \mid \Phi_{i j} \simeq \mathbf{Z}_{m_{i} / d}\right\},
$$

which may be infinite. We set $r_{i}\left(m_{i}\right)=\infty$. Choose $r_{i}(d)$ copies of $B_{i}(d)$ 's; cf. (2.2.2). The objective is to construct a diagram $X$ with these building blocks so that $\mathbf{X}$ has infinite genus and $\partial \mathbf{X}$ is connected and non-compact. Using all $\mathbf{B}_{1}(*)$ 's and some of the $\mathbf{B}_{2}(*)$ 's construct a complex $\mathbf{H}$ homeomorphic to the closed upper half space so that $\partial \mathbf{H}$ contains infinitely many pairs of half-outlets. (Note. That $\mathbf{H}$ contains infinitely many pairs of half-outlets is obvious for $n \geq 3$. For $n=2$ this would fail exactly when $m_{1}=2=m_{2}$. We have explicitly excluded this case in (4.0).) Now attach the remaining building blocks appropriately at these half-outlets so as to get $\mathbf{X}$ with the required properties. 
(4.2) Proposition. If $\Phi$ with $r<\infty$ is realizable as a Neumann subgroup then $r$ is an even integer.

Proof. Indeed, $F_{r} \approx \pi_{1}\left(\mathbf{X}_{\Phi}\right)$. If $S$ is a characteristic subsurface, we observed in $(2.7),(2.8)$ that $\pi_{1}(S) \approx \pi_{1}\left(\mathbf{X}_{\Phi}\right)$ and $S$ is a compact orientable surface with one boundary component. So $r=2 g$ where $g$ is a genus of $S$.

(4.3) Proposition. Let $\Phi$ with $r=2 g<\infty$ be realizable as a Neumann subgroup. Then either

(1) $r_{i}(1)=\infty$ for $\geq n-1$ values of $i$, or,

(2) (A) $r_{i}(1)=\infty, r_{i}(d)<\infty$ for $n-2$ values of $i \neq a, b$, say,

(B) $r_{i}(2)=\infty, r_{i}(d)<\infty, d \neq 2$ for $i=a, b$,

(C) $\Phi$ is a simple $\left(m_{a}, m_{b}\right)$-unfolding of a 1-cycloidal subgroup; cf. (2.11).

Proof. Let $S_{1} \subset S_{2} \subset \cdots$ be an exhaustion of $\mathbf{X}_{\Phi}$ by characteristic subsurfaces. Let $D_{k}=S_{k+1}-$ int $S_{k}, k=1,2, \ldots$ As observed in (2.10), $D_{k}$ is a closed disk and

(4.3.1) $\partial D_{k}=\left\{D_{k} \cap \partial \mathbf{X}_{\Phi}\right\} \cup\left\{\right.$ the two pairs of half-outlets in $\left.\partial S_{k} \cup \partial S_{k+1}\right\}$.

The projection of $D_{k}$ in $Y_{\Phi}$ has the following two possible forms.
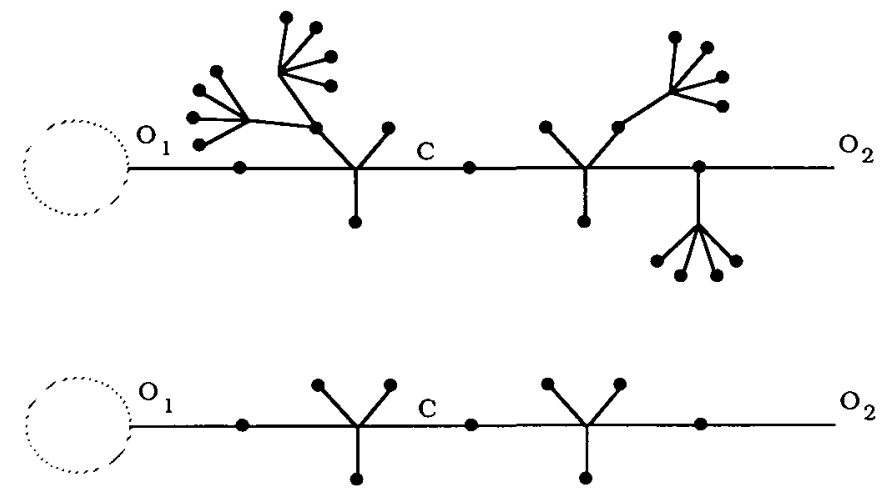

Here $O_{1}, O_{2}$ are the projections of the pairs of half-outlets in $\partial D_{k}$ and $C$ is the shortest path joining $O_{1}$ to $O_{2}$. (Since $D_{k}$ is homeomorphic to a closed disk, $C$ is unique.) The large dark vertices are in $\bigcup \alpha_{i}, i \geq 1$, and the small ones are in $\alpha_{0}$. The two forms are distinguished by the following fact. In (4.3.3) all vertices in $\alpha_{0}$ lie on $C$-hence each is subterminal (cf. (2.3)) and is incident with $n-2$ terminal vertices. In (4.3.2) there are some vertices in $\alpha_{0}$ which do not lie on $C$, and so there are some subterminal ones among 
them which are incident with $n-1$ terminal vertices. Now each terminal vertex is an image of a $B_{i}(1)$ and hence contributes to $r_{i}(1)$. So it follows that $r_{i}(1)=\infty$ for at least $n-2$ values of $i$. Suppose if possible that there actually exist two distinct values $a, b$ of $i$ such that $r_{a}(1)<\infty, r_{b}(1)<\infty$. Then the infinitely many building blocks $\mathbf{B}_{a}(1)$ 's and $\mathbf{B}_{b}(1)$ 's are contained in some characteristic subsurfaces $S_{k_{0}}$. But then for $k>k_{0}, D_{k}$ is necessarily of the form (4.3.3) and the building blocks with two arms in $D_{k}$ are necessarily $\mathbf{B}_{a}(2)$ 's and $\mathbf{B}_{b}(2)$ 's. Since $X_{k_{0}}$ is compact it follows that $r_{i}(d)<\infty$ for $d \neq 1$, $i \neq a, b$, as well. Finally the discussion in (2.11) shows that in this case $\Phi$ must be an $\left(m_{a}, m_{b}\right)$-unfolding of a suitable 1-cycloidal subgroup.

(4.4) Proposition. Let $r=2 g<\infty$, and suppose $r_{i}(1)=\infty$ for at least $n-1$ values of $i$. Then $\Phi$ is realizable as a Neumann subgroup.

Proof. Suppose $r_{i}(1)=\infty$ for $i \neq 1$. The objective is to construct a diagram $X$ with $r_{i}(d)$ copies of $B_{i}(d)$ 's, $d<m_{i}$, and any (possibly infinite) number of copies of $B_{i}\left(m_{i}\right)$ 's so that the thickening diagram $\mathbf{X}$ is an orientable surface of genus $g$ with $\partial \mathbf{X}$ connected and noncompact. Now using finitely many $B_{i}(d)$ 's we can clearly construct a complex $S$ whose thickening $\mathbf{S}$ is a compact, orientable surface of genus $g$ such that $\partial \mathbf{S}$ is connected. (Note. if $\left(m_{1}, m_{2}\right) \neq(2.2)$ or if $g=0$ we can do this using only $B_{i}(d)$ 's, $i \leq 2$. Otherwise we shall need to use $B_{i}(d)$ 's, $i \leq 3$. Here again we are using the assumption that $\Gamma \not \mathbf{Z}_{2} * \mathbf{Z}_{2}$.) Now using all the remaining $B_{1}(d)$ 's and $B_{i}(f)$ 's, $i \geq 2, f \neq 1$, construct a connected complex $V$ whose thickening $\mathbf{V}$ is an orientable surface of genus $g$ such that $\partial \mathbf{V}$ is connected, and contains infinitely many pairs of half-outlets where the infinitely many $\mathbf{B}_{i}(1)$ 's, $i \geq 2$, can be inserted to form $\mathbf{X}$. Clearly $\partial \mathbf{X}$ is connected and $\mathbf{X}=\mathbf{X}_{\Psi}$ where $\Psi$ is a Neumann subgroup isomorphic to $\Phi$.

(4.5) Combining (2.11), (4.1)-(4.4), we get the following

Structure Theorem. Let $\Gamma$ be as in (1.4.1), $\Gamma \not \equiv \mathbf{Z}_{2} * \mathbf{Z}_{2}$ and $\Phi$ be given as an abstract group as in (1.7.1). Then $\Phi$ is realizable as a Neumann subgroup of $\Gamma$ if and only if one of the following three conditions holds:

(1) $r=\infty$;

(2) (A) $r$ is an even non-negative integer,

(B) $r_{i}(1)=\infty$ for $\geq n-1$ values of $i$;

(3) (A) $r$ is an even non-negative integer,

(B) $r_{i}(1)=\infty, r_{i}(d)<\infty, d \neq 1$, for $n-2$ values of $i \neq a, b$ say,

(C) $r_{i}(2)=\infty, r_{i}(d)<\infty, d \neq 2$ for $i=a, b$,

(D) there exists $\Phi_{0}$, realizable as a 1-cycloidal subgroup such that $\Phi$ is a simple $\left(m_{a}, m_{b}\right)$-unfolding of $\Phi_{0}$. 
(4.6) Remark. Suppose $\Phi$ is as in (1.7.1) and (3) (A)-(C) are satisfied. Let $\Psi_{0}$ be the finite free product of $F_{r}$ and $\Phi_{i j} \approx \mathbf{Z}_{m_{i} / d}, i \neq a, b$ and $d \neq 1$, or $i=a, b, d \neq 2$. If $\Phi$ is realizable as a Neumann subgroup then $\Phi_{0}$ referred to in (3) (D) is isomorphic to $\Psi_{0} * \boldsymbol{\theta}_{0}$ where $\boldsymbol{\theta}_{0}$ is a finite free product of groups conjugate to $\Gamma_{i}, i \neq a, b$, or conjugate to the subgroups of $\Gamma_{a}$ (respectively $\Gamma_{b}$ ) isomorphic to $\mathbf{Z}_{a / 2}$ (respectively $\mathbf{Z}_{b / 2}$ ). Moreover $\Phi_{0}$ must contain at least one factor isomorphic to $\Gamma_{a}$ or $\Gamma_{b}$. From the way $X_{\Phi_{0}}$ would be constructed (cf. (2.11)) it is clear that there are only finitely many possibilities for $\boldsymbol{\theta}_{0}$, and hence, also only finitely many possibilities for $\Phi_{0}$. Now [5, Theorem (1.5)] gives an effective procedure for deciding whether any of these $\Phi_{0}$ can be realized as a 1-cycloidal subgroup. Thus one has an effective procedure for deciding realizability of $\Phi$ as a Neumann subgroup.

(4.7) Remark. The condition (3) (D) is not a consequence of (3)(A)-(C). For example, take $\Gamma=\mathbf{Z}_{4} * \mathbf{Z}_{4}$ and $\Phi=\Pi^{*} \mathbf{Z}_{2}$ (infinite product). Write $\Phi$ as

$$
\prod_{i=1}^{* 2}\left(\prod_{j \in J_{i}}^{*} \boldsymbol{\Phi}_{i j}\right), \quad \Phi_{i j} \approx \mathbb{Z}_{2}, \quad\left|J_{i}\right|=\infty .
$$

It is easy to see that $(3)(A)-(C)$ hold, but $\Phi$ is not realizable as a Neumann subgroup.

(4.8) Remark. We should point out two possible interpretations for the phrase " $\Phi$ as in (1.7.1) is realizable as...". If $m_{i}$ 's are pairwise coprime then there is a unique value of $i$ for a finite factor of $\Phi$ to be conjugate to a subgroup of $\Gamma_{i}$. If two or more $m_{i}$ 's have common factors then there may be a choice for a finite factor of $\Phi$ to be interpreted as a particular $\Phi_{i j}$. In our statement of the structure theorem we have tacitly assumed that these choices have already been made. Thus if $\Phi$ is only given as an abstract group there may be a bit more freedom first to put it in the form (1.7.1) and then realize as a....

(4.9) REMARK. The condition (3)(C) of course requires that $m_{a}$ and $m_{b}$ are even integers. So if there is at most one $M_{i}$ which is an even integer then the condition (3) is not applicable.

\section{Maximal subgroups}

(5.0) In [3] and [13] there are constructions of subgroups of the classical modular group which are maximal among nonparabolic subgroups, and which are different from the ones discovered by Neumann [8], or which are not 
Neumann subgroups in the sense of (1.1). These constructions are rather elaborate and require a very careful analysis. In terms of the diagrams $X_{\Phi}$ 's one can give such constructions more readily, and in fact one may construct maximal, or maximal and Neumann, or maximal and 1-cycloidal, or maximal and non-parabolic but not Neumann... subgroups.

(5.1) Let $\Gamma$ be as in (1.4.1) and $\Phi \leq \Gamma$. A symmetry of $\mathbf{X}_{\Phi}$ is simply a branched-covering-transformation of $p: \mathbf{X}_{\Phi} \rightarrow \mathbf{X}_{\Gamma}$, that is, a homeomorphism $\sigma: \mathbf{X}_{\Phi} \rightarrow \mathbf{X}_{\Phi}$ such that

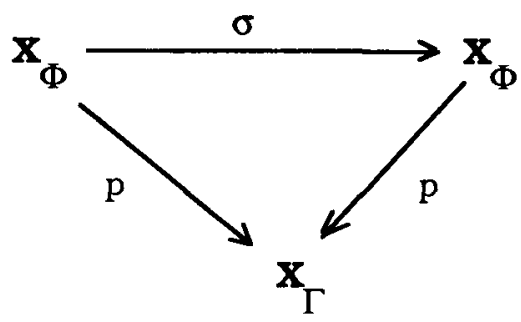

commutes. Then $\sigma$ preserves orientation and carries building blocks into building blocks.

Notice that in an unbranched covering space a non-identity covering transformation has no fixed points. But in a branched covering it is not necessarily so.

We say that $\mathbf{X}_{\boldsymbol{\Phi}}$ has no fixed-point-free symmetry if every non-identity symmetry of $\mathbf{X}_{\Phi}$ has a fixed point.

Notice also that a symmetry $\sigma: \mathbf{X}_{\Phi} \rightarrow \mathbf{X}_{\Phi}$ induces maps (again denoted by) $\sigma: X_{\Phi} \rightarrow X_{\Phi}$ and $\sigma: Y_{\Phi} \rightarrow Y_{\Phi}$, and these maps commute with the thinning map and the canonical projection $X_{\Phi} \rightarrow Y_{\Phi}$.

(5.2) Orient $\mathbf{X}_{\Phi}$; this also orients $\partial \mathbf{X}_{\Phi}$. Let $C$ be a component of $\partial \mathbf{X}_{\Phi}$. The pattern along $C$ is simply the finite or doubly infinite sequence of $\mathbf{B}_{i}(d)$ 's one meets along $C$ while walking in the "positive" direction. Notice that a block $\mathbf{B}_{i}(d)$ with $d>1$ (see the picture in (2.3)), is counted $k$ times in the patterns along $C$ if $C$ contains $k$ "circular arcs" on $\mathbf{B}_{i}(d)$, that is, the components $\partial \mathbf{B}_{i}(d)-\partial\{$ Uarms $\}$.

The pattern is finite if and only if $C$ is compact and in that case the number of terms in the pattern is a multiple of $n$. We say that the pattern along $C$ is not periodic if either (1) $C$ is noncompact and the pattern has no finite period or (2) $C$ is compact, the pattern contains $\alpha n$ elements, $\alpha \in \mathbf{Z}_{>0}$, and (in the cyclic order) the pattern has no period strictly less than $\alpha n$.

(5.3) Let $B=B_{i}(d)$ be a building block of $X_{\Phi}$. The neighbors of $B$ are the building blocks at the end of the paths containing two edges emanating from $B$. So, in all $B$ has $d(n-1)$ neighbors. 
(5.4) THEOREM. Let $\Gamma$ be as in (1.4.1) where all $m_{i}$ 's are primes. Let $\Phi \leq \Gamma$ be as in (1.7.1). Assume that (1) each $B \approx B_{i}\left(m_{i}\right)$ in $X_{\Phi}$ has a $B_{j}\left(m_{j}\right)$ for each $j \neq i$ as a neighbor, and (2) either (a) $r=0$ and $\mathbf{X}_{\Phi}$ has no fixed-point-free symmetry or (b) the patterns along different components of $\partial \mathbf{X}_{\Phi}$ are pairwise distinct and none is periodic. Then $\Phi$ is maximal.

Proof. Suppose $\Phi \leq \Psi \leq \Gamma$, and consider the branched covering $q: \mathbf{X}_{\Phi} \rightarrow$ $\mathbf{X}_{\Psi}$. Suppose $\mathbf{X}_{\Psi}$ contains a branch point. Since $m_{i}$ 's are assumed to be primes this means that there is a building block $B \subseteq X_{\Phi}$ such that $B \approx B_{i}\left(m_{i}\right)$ and $q(B) \approx B_{i}(1)$. But then (1) implies that $q\left(X_{\Phi}\right)=X_{\Gamma}$, that is, $\Psi=\Gamma$.

Now suppose $\Psi \neq \Gamma$. Hence $q$ is unbranched. Under the condition (2a), $\mathbf{X}_{\Phi}$ is simply connected. But then $q$ is the universal (in particular regular) covering of $\mathbf{X}_{\Psi}$. Since we assumed that $\mathbf{X}_{\Phi}$ has no fixed-point-free symmetry it follows that degree $q=1$, and $\Phi=\Psi$. Under the condition (2b) we see that $\left.q\right|_{\partial \mathbf{X}_{\Phi}}$ is a homeomorphism. Also clearly $q^{-1}\left(\partial \mathbf{X}_{\Psi}\right)=\partial \mathbf{X}_{\Phi}$. So again degree $q=$ degree $\left.q\right|_{\partial \mathrm{x}_{\Phi}}=1$ and $\Phi=\Psi$. Hence $\Phi$ is maximal.

(5.5) Remarks. (1) Clearly there are many varieties of sufficient sets of conditions for maximality in terms of $X_{\Phi}$ 's. For instance one may assume that all but finitely many building blocks of $X_{\Phi}$ have the property stated in (1) and then "mess up" the diagram near these finitely many blocks.

(2) If $n \geq 2$ or two $m_{i}$ 's $\geq 3$ the conditions in (5.4) are easy to ensure. The condition that $\mathbf{X}_{\Phi}$ has no fixed-point symmetry is ensured if we have a compact subsurface $S \subseteq X_{\Phi}$ satisfying the condition (2.8.1) such that $S$ is homeomorphic to a closed disk and the pattern of the building blocks in $S$ does not repeat in $X_{\Phi}$, or at least the "distances" among its repetitions do not repeat. Then any symmetry of $\mathbf{X}_{\Phi}$ would leave $S$ invariant and would have a fixed point by Brouwer's theorem.

(3) If $n=2$ and some $m_{i}=2$ then the direct application of (5.4) produces only finitely many examples, all of finite index. But excluding the degenerate case $\Gamma \approx \mathbf{Z}_{2} * \mathbf{Z}_{2}$ one may first pass to an appropriate 1-cycloidal subgroup in $\Gamma$ and then apply the above considerations. For example let $\Gamma=\mathbf{Z}_{2} * \mathbf{Z}_{3}$, and let $\Phi_{0} \subset \Gamma, \Phi_{0} \approx \mathbf{Z}_{3} * \mathbf{Z}_{3}$ whose diagram is

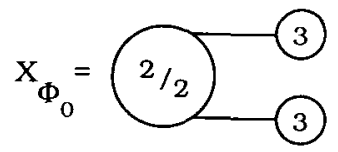

$(5.5 .1)$

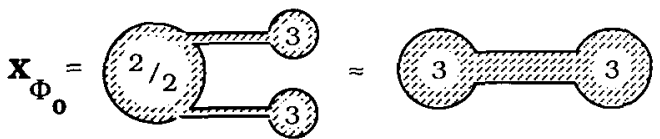


Consider $\Phi \subset \Phi_{0}$ whose diagram is

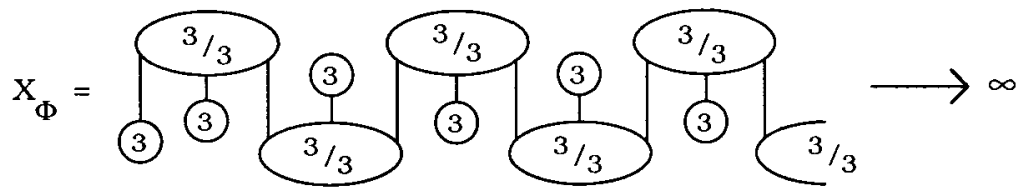

Clearly $\Phi$ is a Neumann subgroup of $\Phi_{0}$, and in fact maximal in $\Phi$. Also clearly $\Phi$ is a Neumann subgroup of $\Gamma$. As a subgroup $\Gamma$, the diagram of $\Phi$ is obtained from (5.5.2) by sticking in $2 / 2$ on each edge. If we do this sticking and then replace one - (3/2) by - (2) we obtain a new Neumann subgroup of $\Gamma$ which is clearly not contained in $\Phi_{0}$. It would be also maximal in $\Gamma$. Making the pattern in (5.52) doubly infinite in the obvious way one obtains a subgroup $\Phi_{1} \subset \Phi_{0}$ for which $\partial \mathbf{X}_{\Phi_{1}}$ contains two components both noncompact. This $\Phi_{1}$ is not Neumann and it is maximal among nonparabolic subgroups in $\Phi_{0}$ and also in $\Gamma$ but it is not maximal. For clearly $\Phi_{1} \leq \Psi_{1} \leq \Phi_{0}$, where

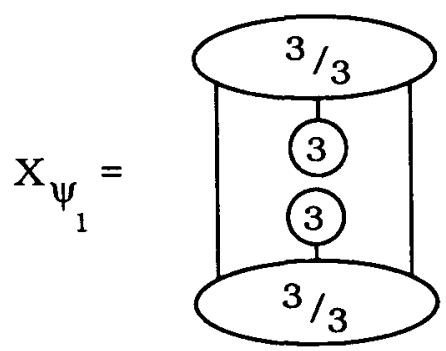

so $\Phi_{1}$ is not maximal. On the other hand if $\Phi_{1}<\Psi<\Phi_{0}$, then $q$ : $\mathbf{X}_{\Phi_{1}} \rightarrow \mathbf{X}_{\Psi}$ must be unbranched; see the argument in (5.4). Now $\mathbf{X}_{\Phi_{1}}$ is simply connected so $q$ must be a regular covering. One sees that the only symmetries of $\mathbf{X}_{\Phi_{1}}$ are the obvious "horizontal" translations, and so $\mathbf{X}_{\Psi}$ is compact, that is, $\left(\Phi_{0}: \Psi\right)<\infty$. So $\Psi$ contains parabolic elements. Thus $\Phi_{1}$ is maximal among non-parabolic subgroups. On the other hand one may start with a doubly infinite version of (5.5.2) where the attachment of (3) non-periodic. Then one would obtain a maximal-and-non-parabolic subgroup of $\Phi_{0}$ which is not Neumann. By sticking in - (2) somewhere (as described above) one would obtain such subgroups also in $\Gamma$. 


\section{References}

[1] J. L. Brenner and R. C. Lyndon, 'Nonparabolic subgroups of the modular group', J. Algebra 77 (1982), 311-322.

[2] J. L. Brenner and R. C. Lyndon, 'Permutations and cubic graphs', Pacific J. Math. 104 (1983), 285-315.

[3] J. L. Brenner and R. C. Lyndon, 'Maximal nonparabolic subgroups of the modular group', Math. Ann. 263 (1983), 1-11.

[4] R. S. Kulkarni, 'An extension of a theorem of Kurosh and applications to Fuchsian groups', Michigan Math. J. 30 (1983), 259-272.

[5] R. S. Kulkarni, 'Geometry of free products', Math. Z. 193 (1986), 613-624.

[6] W. Magnus, 'Rational representations of Fuchsian groups and nonparabolic subgroups of the modular group', Nachr. Akad. Wiss. Göttingen Math.-Phys. Kl. II 9 (1973), 179-189.

[7] W. Magnus, Noneuclidean tesselations and their groups, (Academic Press, New York, 1974).

[8] B. H. Neumann, 'Über ein gruppen-theoretisch-arithmetisches problem', Sitzungsber. Preuss. Akad. Wiss. Math.-Phys. Kl. 10 (1933).

[9] H. Petersson, 'Über einen einfachen typus von untergurppen der modulgruppe', Arch. Math. 4 (1953), 308-315.

[10-12] W. W. Stothers, 'Subgroups of infinite index in the modular group I-III', Glasgow Math. J. 20 (1979), 103-114, ibid. 22 (1981), 101-118, ibid. 22 (1981), 119-131.

[13] C. Tretkoff, 'Nonparabolic subgroups of the modular group', Glasgow Math. J. 16 (1975), 90-102.

Department of Mathematics

City University of New York

33 W. 42nd Street

New York, New York 10036-8099

U.S.A. 